\title{
Effects of weight training time on bone mineral density of patients with secondary osteoporosis after hemiplegia
}

\author{
LIANG HAN ${ }^{*}$, SHU-GANG LI* , HONG-WEI ZHAI, PENG-FEI GUO and WEI CHEN \\ Department of Rehabilitation, Xuzhou Central Hospital, Xuzhou, Jiangsu 221009, P.R. China
}

Received February 10, 2016; Accepted November 11, 2016

DOI: $10.3892 /$ etm.2017.4078

\begin{abstract}
The aim of the study was to investigate the effects of weight training time on bone mineral density (BMD) of patients with secondary osteoporosis after hemiplegia. A total of 129 hemiplegic patients with stroke, 75 males and 54 females, aged 50-75 years, were recruited in this study. Male patients were randomly divided into 3 groups ( 25 cases each) receiving 30 min (group M30), 60 min (group M60), or 90 min (group M90) daily upright bed weight training. Female patients were similarly randomly allocated into groups F30, F60 and F90 (18 cases each) with daily upright bed weight training for 30, 60 and 90 min, respectively. After 3 months of training, BMD at anteroposterior lumbar spine (L1-L4) and ipsilateral femoral neck were examined by dual-energy X-ray absorptiometry. Compared to pre-treatment, no statistical difference in BMD was found after 3 months in males who underwent daily 30-min weight training (group M30). By contrast, a significant increase in BMD was seen at lumbar and femoral neck in groups M60 and M90 after 3 months (all p<0.05). By pair-wise comparison, we found that BMD at lumbar and at femoral neck was significantly higher in groups M60 vs. M30 and in groups M90 vs. M60 (all p<0.05). For female subjects, daily 90-min weight training significantly increased BMD at lumbar and at femoral neck in group F90 (both p<0.05), but daily 30- or 60-min weight training did not produce similar improvement after 3 months. By pair-wise comparison, we found a significantly higher BMD at lumbar and at femoral neck in groups F90 vs. F60 after 3 months of treatment (both $\mathrm{p}<0.05$ ). In conclusion, in order to stimulate BMD and reverse osteoporosis in patients with secondary osteoporosis after hemiplegia, daily weight training for minimum 60 and $90 \mathrm{~min}$ was needed for males and females, respectively.
\end{abstract}

Correspondence to: Dr Wei Chen, Department of Rehabilitation, Xuzhou Central Hospital, 199 Jiefang South Road, Xuzhou, Jiangsu 221009, P.R. China

E-mail: ctqabe3@163.com

*Contributed equally

Key words: stroke, hemiplegia, osteoporosis, bone mineral density, weight training

\section{Introduction}

Osteoporosis is a significant complication of stroke, associated with an increased incidence of fractures, leading to further disability (1-4). The pattern of bone loss seen in stroke patients is different from that usually encountered with postmenopausal osteoporosis, since it is limited to the hemiplegic limb. The clinical course of hemiplegic stroke predisposes patients to disturbed bone physiology. Sudden immobility and unilateral loss of function unload the skeleton at key areas such as the affected hip. This is manifest by an early reduction in bone density at this site (5-8).

Several factors appear to have an influence on bone mass in stroke patients, such as the degree of paresis, gait disability, the duration of immobilization, serum level of vitamin D and gender $(9,10)$. Although recovery of brain functions and ability of independent movement in stroke patients has achieved significant success in recent years, potential bone loss and secondary osteoporosis are often overlooked in stroke treatment before fragility fracture occurred (10).

In addition to drug therapy, rehabilitation training plays an important role in preventing and managing osteoporosis after stroke. Currently, consensus on proper modes of movement and exercise prescription of specific rehabilitation training is lacking. Our study focused on weight training and aimed to investigate the effects of training time on bone mineral density (BMD) of patients with secondary osteoporosis after hemiplegia. Results from the present study provide some clinical evidence on how a specific rehabilitation program can prevent and better manage osteopenia and osteoporosis after stroke.

\section{Materials and methods}

Study participants and patient allocation. The present study was approved by the Ethics Committee of Xuzhou Central Hospital (Jiangsu, China). All the patients or their relatives signed an informed consent prior to their participation.

A total of 129 stroke patients who were hospitalized in the Department of Rehabilitation, Central Hospital of Xuzhou, from February 2013 to February 2015 were enrolled in this study. They comprised 75 males, aged 50-75 years (mean age of 65.34 \pm 5.26 years), and 54 females, also aged 50-75 years (mean age of $68.45 \pm 6.54$ years). Inclusion criteria for the study were: i) Patients conformed to the diagnostic criteria set in the fourth National Conference on Diagnosis of Stroke, 
Table I. Patient information and pathogenetic conditions.

\begin{tabular}{lcccccc}
\hline & & & & & & \multicolumn{2}{c}{ Stroke type (cases) } \\
\cline { 6 - 7 } Group & Cases & Gender & $\begin{array}{c}\text { Age (years, } \\
\text { mean } \pm \text { SD) }\end{array}$ & $\begin{array}{c}\text { Course of disease } \\
\text { (months, mean } \pm \text { SD) }\end{array}$ & Cerebral infarction & Cerebral hemorrhage \\
\hline M30 & 25 & M & $65.4 \pm 5.72$ & $5.6 \pm 1.58$ & 19 & 6 \\
M60 & 25 & M & $62.7 \pm 4.25$ & $5.7 \pm 1.76$ & 18 & 7 \\
M90 & 25 & M & $67.9 \pm 5.68$ & $5.3 \pm 1.64$ & 19 & 6 \\
F30 & 18 & F & $66.3 \pm 4.82$ & $4.7 \pm 1.32$ & 14 & 4 \\
F60 & 18 & F & $70.2 \pm 7.69$ & $4.4 \pm 1.43$ & 12 & 6 \\
F90 & 18 & F & $68.8 \pm 6.27$ & $4.6 \pm 1.54$ & 13 & 5 \\
\hline
\end{tabular}

M, male; F, female.

and confirmed diagnosis with stroke by head CT or MRI; ii) $\geq 50$ years of age, $\leq 75$ years; and iii) agreed to take BMD measurements on the femoral neck of the affected limb, $\mathrm{T} \leq 1$ (BMD was presented by T-score $(\mathrm{T})$, where $\mathrm{T}=$ (measured value-peak bone mass)/BMD standard deviation (SD) of normal people (11). Exclusion criteria for the study were: i) Patients with severe heart, liver and kidney diseases, severe diabetics, and other organic diseases in endocrinium; ii) patients had taken drugs that would seriously affect the bone metabolism three months before onset; iii) patients that were lost to follow up; iv) patients that failed to complete all of the inspections and treatments; and v) whose consent forms were not obtained from the patients or relatives.

Diagnosis of secondary osteoporosis in stroke patients was made according to the diagnostic criteria recommended by WHO, and the readings acquired by dual-energy X-ray absorptiometry (DXA). Those with BMD one SD lower than the peak bone mass of a normal adult of the same gender and the same ethnicity were classified as normal. Those with BMD 1-2.5 SD lower than the peak bone mass of a normal adult of the same gender and the same ethnicity were defined as osteopenia. Those with BMD higher than 2.5 SD were classified as osteoporosis. Those with decreased BMD to the standard level of osteoporosis, accompanied with $\geq 1$ fractures were defined as severe osteoporosis (11).

Patient allocation was performed using the random number table method. Male patients were divided into 3 groups: M30, M60 and M90, 25 cases each. They received daily 30-, 60- and 90-min upright bed weight training, respectively. Female patients were similarly allocated into groups F30, F60 and F90 (18 cases each) with daily upright bed weight training for 30, 60 and $90 \mathrm{~min}$, respectively. Differences in general and pathogenetic conditions between the groups of the same gender were not statistically significant ( $>0.05$; Table I).

Treatment methods. All the patients received basic treatment which included: i) life style adjustment: balanced diet with abundant calcium, proper protein and low intake of salt; appropriate outdoor activities and sunshine; no smoking or drinking; proactive methods to prevent from falling; and ii) basic dietary supplements for bone health: oral calcium carbonate D3 (state approval no. H10950029), one pill/time, twice per day, equivalent to $1,200 \mathrm{mg} /$ day calcium and
250 IU/day vitamin D3. Additionally, daily standing bed weight training was provided to patients in groups M30 and F30 for $30 \mathrm{~min}$, groups M60 and F60 for $60 \mathrm{~min}$, and groups M90 and F90 for $90 \mathrm{~min}$. Specifically, the patients in $\mathrm{M} 30 / \mathrm{F} 30$ were required to take weight training for $30 \mathrm{~min}$, once per day (at 8:30 a.m.). Patients in groups M60/F60 were required to take weight training twice per day (at 8:30 a.m. and 2:30 p.m. with an interval of $6 \mathrm{~h}$ ). Patients in groups M90/F90 were required to take weight training three times per day at 8:30 a.m., 11:30 a.m. and 2:30 p.m., with an interval of $3 \mathrm{~h}$. All the patients underwent weight training 5 days/week for 3 months.

Measurement method. BMD at anteroposterior lumbar spine (L1-L4) and ipsilateral femoral neck were examined by using Norland XR-600 dual X-ray rapid entire body BMD tester before and 3 months after weight training.

Statistical analysis. SPSS 16.0 statistical software (SPSS, Inc., Chicago, IL, USA) was applied to process the data. All data were presented as mean \pm SD. Normal distribution and homoscedasticity were examined and noted. Student's t-test was used to compare BMD before and after treatment within groups. Comparison between groups was evaluated by single factor variance analysis. Least significant difference (LSD) was applied to pair-wise comparisons between sub-groups. $\mathrm{P}<0.05$ was considered statistically significant.

\section{Results}

In our study, the number of patients who had recovered walking ability under assistance was recorded after rehabilitation training for 1-3 months. For males, 36\% (9/25 cases) in group M30, 40\% (10/25) in group M60 and 28\% (7/25) in group M90 had recovered walking ability. The numbers for females were 33\% (6/18 cases) in group F30, 33\% (6/18 cases), and $27.8 \%$ (5/18) in group F90. Seeing that the aim of early rehabilitation of training was focused on the patients' gait, thus therapists recommended that patients should stand and walk for $\leq 30 \mathrm{~min}$ after their ability to stand alone or walk with assistance was recovered. In this way, patients could avoid developing abnormal gaits and we could guarantee the comparability of patients on weight training time. 
Table II. Comparison of BMD in affected limbs of hemiplegic male patients before and after training treatment $\left(\mathrm{g} / \mathrm{cm}^{2}, \mathrm{mean} \pm \mathrm{SD}\right.$, $\mathrm{N}=25$ in each group).

\begin{tabular}{lcccc}
\hline & \multicolumn{2}{c}{ Before training } & \multicolumn{2}{c}{ After training } \\
\cline { 2 - 3 } Group & Lumbar vertebra & Femoral neck & & Lumbar vertebra \\
\hline M30 & $0.808 \pm 0.146$ & $0.719 \pm 0.108$ & $0.810 \pm 0.124^{\mathrm{a}}$ & $0.721 \pm 0.107^{\mathrm{a}}$ \\
M60 & $0.819 \pm 0.133$ & $0.720 \pm 0.124$ & $0.906 \pm 0.137^{\mathrm{b}}$ & $0.785 \pm 0.118^{\mathrm{c}}$ \\
M90 & $0.817 \pm 0.137$ & $0.715 \pm 0.116$ & $0.971 \pm 0.142^{\mathrm{b}, \mathrm{d}}$ & $0.851 \pm 0.122^{\mathrm{a}, \mathrm{b}}$ \\
\hline
\end{tabular}

${ }^{\text {aP }}<0.01$ compared to group M60 after training; ${ }^{\text {b }} \mathrm{P}<0.01$ compared to before training within groups (M60 and $\mathrm{M} 90$ ); ${ }^{\mathrm{c}} \mathrm{P}<0.05$ compared to before training in group M60; ${ }^{\mathrm{P}}<0.05$ compared to group M60 after training. BMD, bone mineral density.

Table III. Comparisons of BMD in affected limbs of hemiplegic female patients before and after treatment $\left(\mathrm{g} / \mathrm{cm}^{2}, \pm \mathrm{s}, \mathrm{N}=18\right.$ in each group).

\begin{tabular}{|c|c|c|c|c|}
\hline \multirow[b]{2}{*}{ Group } & \multicolumn{2}{|c|}{ Before training } & \multicolumn{2}{|c|}{ After training } \\
\hline & Lumbar vertebra & Femoral neck & Lumbar vertebra & Femoral neck \\
\hline F30 & $0.729 \pm 0.119$ & $0.674 \pm 0.112$ & $0.732 \pm 0.105$ & $0.677 \pm 0.125$ \\
\hline F60 & $0.718 \pm 0.131$ & $0.668 \pm 0.118$ & $0.726 \pm 0.129$ & $0.679 \pm 0.113$ \\
\hline F90 & $0.726 \pm 0.128$ & $0.681 \pm 0.122$ & $0.805 \pm 0.117^{\mathrm{a}, \mathrm{b}}$ & $0.743 \pm 0.128^{\mathrm{a}, \mathrm{b}}$ \\
\hline
\end{tabular}

${ }^{\mathrm{a}} \mathrm{P}<0.05$ compared to group $\mathrm{F} 90$ before training; ${ }^{\mathrm{b}} \mathrm{P}<0.05$ compared to group $\mathrm{F} 60$ after training. BMD, bone mineral density.

Data of BMD at lumbar vertebra and femoral neck of the affected limb were taken from all the study participants and tabulated in Tables II and III. Compared to pre-treatment, no statistical difference in BMD was found after 3 months in males who underwent daily 30-min weight training (group M30; Table II). By contrast, a significant increase in BMD was seen at lumbar vertebra and femoral neck in groups M60 and M90 after 3 months (all p<0.05). Results from pair-wise comparison showed that BMD at lumbar vertebra and at femoral neck was significantly higher in groups M60 vs. M30 (both p<0.01) and in groups M90 vs. M60 (p<0.05 and p<0.01, respectively).

For female subjects, daily 90-min weight training significantly increased BMD at lumbar and at femoral neck in group F90 (both p<0.05), but daily 30-min or 60-min weight training did not produce significant BMD improvement after 3 months (Table III). By pair-wise comparison, we found a significantly higher BMD at lumbar and at femoral neck in group F90 vs. group F60 after 3 months of treatment (both $\mathrm{p}<0.05)$.

\section{Discussion}

Considering the particularity of stroke patients' functional disturbances, dynamic standing bed was selected as the weight training method, which required the two lower extremities to bear the same weight. After overcoming postural hypotension, patients in groups M30/F30, M60/F60 and M90/F90 were required to take weight training for $30 \mathrm{~min}$, once a day, twice a day, or three times a day, respectively, 5 days/week for 3 months.
Stroke patients, being affected by immobilization resulting from early movement function limitations, usually had disordered calcium metabolism and insufficient vitamin D, which further led to disuse osteoporosis. Chung et al showed that one week after the infarction of middle cerebral artery, SpragueDawley rats started to develop osteoclastogenesis and increased differentiation, which would further lead to increased bone resorption (12). Lin et al demonstrated that secondary osteoporosis that occurred in the first four years after stroke was an important risk factor of hip fracture. They also advocated that early treatment of stroke should be focused on early prevention of osteopenia (13). Another study on osteoporosis revealed that low bone density in femoral neck and osteoporosis would result in increasing stroke risks and mortality (14). A prospective study by European researchers also showed that osteoporosis may directly increase the incidence of stroke (15). All of these studies have suggested that stroke and osteoporosis were in reciprocal causation. If proper intervention was not made, both may be trapped in a vicious cycle.

Although the risks of fracture on patients with secondary osteoporosis after stroke would increase as BMD decreased, most of the osteoporotic fractures occurred on osteopenia patients. Albeit the fracture risk of osteopenia patients was lower than that of the osteoporosis patients, the number of osteopenia patients was far more than the osteoporosis patients according to WHO diagnostic criteria and on the basis of normal distribution principle (16). Thus, in our study, we chose patients with osteopenia and osteoporosis, and with BMD T-score $<-1$, for our study subjects.

Some systematic reviews and RCT studies have confirmed that rehabilitation training after stroke could significantly 
reduce the incidence of secondary osteoporosis and the risk of fracture. Effective intervention methods included: skeleton weight training and muscular force training $(17,18)$. According to Wolff's law, skeleton had the mechanical ability to adjust to the bone tissue during activities. Different weight training methods were conductive to the bone health of elderly individuals. Weight training, through the direct addition of mechanical stimulation, could activate osteoblast, promote the mineral substances to deposit in the stress points of bone, increase BMD, and stimulate the formation of bone $(19,20)$. In our study, on the basis of clinical practice of stroke patients, we implemented dynamic standing bed weight training to study its influence on the bone loss of post-stroke secondary osteoporosis patients. Moreover, considering that gender had a great influence on the degree of osteoporosis, we also divided the patients into the male and female groups randomly so that we could effectively control the disturbance and influence of non-experimental factors, including gender, age, course of disease and illness degree, on the final results.

Weight training time was made on the premise that patients could not stand or walk because of dyskinesia. With the intervention of systematic rehabilitation training, certain patients could walk with assistance (that is, they could walk a short distance indoor under the assistance of another person, mobility aids or quadropod). However, the differences in their walking recovery time and degree were considerably large, thus we could not confirm the active weight bearing time of the patients.

A series of studies have shown that early rehabilitation training after stroke could effectively prevent secondary osteoporosis and other complications, but none of these studies focused on the training methods and training prescriptions $(9,10)$. Guidance on the Diagnosis and Treatment of Primary Osteoporosis has pointed out that therapy, which was focused on kinesitherapy, has been widely popularized (11). Weight-bearing training, resistance training, overload training, and accumulated exercise training could produce osteogenic effects. Modes of exercise included weight-bearing exercise, and resistance training. It was suggested that patients should take weight-bearing training 4 to 5 times a week, and resistance training 2 to 3 times a week. Patients with quadriplegia, paraplegia, and hemiplegia were more liable to secondary osteoporosis because their nerve injuries and muscle losses were more serious. Thus, they had enhanced weight bearing training and resistance training on their limbs. However, consensus on specific exercise frequency and intensity is lacking. Guidance on the Diagnosis and Treatment of Secondary Osteoporosis did not mention the diagnostic or treatment principle on posthemiplegic secondary osteoporosis, but mentioned that besides general therapy and drug therapy, patients with immobilization osteoporosis (disuse osteoporosis) shall pay more attention to exercise and rehabilitation treatment on the immobilization site (21). However, detailed rehabilitation methods and prescriptions were not provided.

In the present study, we used a gradient type method. After weight bearing methods and prescriptions were determined, and the time intensity of weight training was divided into 30 , 60 , and $90 \mathrm{~min}$. After a period of observation, we discovered that the degree of secondary osteoporosis on patients after stroke was greatly improved and that to increase BMD, male patients should keep training for at least $60 \mathrm{~min} / \mathrm{day}$ and female patients should keep training for at least $90 \mathrm{~min} / \mathrm{day}$. The results from the present study provide relatively precise training time intensity for patients to prevent and improve posthemiplegic secondary osteoporosis.

Results from the present study showed that an increase in BMD on the affected limb of hemiplegic patients was closely related with the time intensity of weight training. As weight bearing time went on, BMD on affected limbs increased. But within our study period, we did not observe any platform stage or declining period. How BMD would develop if daily weight training time was extended is unknown. Due to the limited feasibility of rehabilitation and compliance of patients, we did not further extend our study. Nevertheless, further investigation on favorable training methods and course of treatment is required.

In conclusion, previous studies have reported that weight training could improve the BMD of osteoporosis patients. Our study further confirmed the minimum weight training intensity required for patients to increase their BMD. Specifically, male patients should take daily weight training for at least $60 \mathrm{~min}$, and female patients need at least $90 \mathrm{~min}$ of daily weight training. Training intensity lower than these levels may compromise full benefits. These results may provide a basis for clinical practice and be applied to poststroke hemiplegic patients.

\section{References}

1. Golob AL and Laya MB: Osteoporosis: screening, prevention, and management. Med Clin North Am 99: 587-606, 2015.

2. Giusti A and Bianchi G: Treatment of primary osteoporosis in men. Clin Interv Aging 10: 105-115, 2014.

3. Adler RA: Osteoporosis in men: recent progress. Endocrine 44: 40-46, 2013.

4. Zhang J, Morgan SL and Saag KG: Osteopenia: debates and dilemmas. Curr Rheumatol Rep 15: 384, 2013.

5. Pluskiewicz W: Skeletal consequences in patients after stroke. Endokrynol Pol 62: 48-50, 2011.

6. Beaupre GS and Lew HL: Bone-density changes after stroke. Am J Phys Med Rehabil 85: 464-472, 2006.

7. Painter SE, Kleerekoper M and Camacho PM: Secondary osteoporosis: a review of the recent evidence. Endocr Pract 12: 436-445, 2006.

8. Diab DL and Watts NB: Postmenopausal osteoporosis. Curr Opin Endocrinol Diabetes Obes 20: 501-509, 2013.

9. Levis S and Theodore G: Summary of AHRQ's comparative effectiveness review of treatment to prevent fractures in men and women with low bone density or osteoporosis: update of the 2007 report. J Manag Care Pharm 18: S1-S15; discussion S13, 2012.

10. Carda S, Cisari C, Invernizzi M and Bevilacqua M: Osteoporosis after stroke: a review of the causes and potential treatments. Cerebrovasc Dis 28: 191-200, 2009.

11. The Chinese Osteoporosis and bone Mineral Association: Guide for diagnosis and treatment of primary osteoporosis. Chinese $\mathrm{J}$ Osteoporosis Bone Mineral Res 4: 2-17, 2011 (In Chinese).

12. Chung ME, Lee JI, Im S and Park JH: Ischemic stroke in rats enhances bone resorption in vitro. J Korean Med Sci 27: 84-88, 2012.

13. Lin HL, Lin HC, Tseng YF, Liao HH, Worly JA, Pan CY and Hsu CY: Hip fracture after first-ever stroke: a population-based study. Acta Neurol Scand 131: 158-163, 2015.

14. Zhou R, Liu D, Li R, Zhou S, Cui M, Chen L and Zhou H: Low bone mass is associated with stroke in Chinese postmenopausal women: the Chongqing Osteoporosis Study. Cell Biochem Biophys 71: 1695-1701, 2014.

15. Myint PK, Clark AB, Kwok CS, Loke YK, Yeong JK, Luben RN, Wareham NJ and Khaw KT: Bone mineral density and incidence of stroke: European prospective investigation into cancer-norfolk population-based study, systematic review, and meta-analysis. Stroke 45: 373-382, 2014. 
16. Eriksen EF: Treatment of osteopenia. Rev Endocr Metab Disord 13: 209-223, 2012

17. Bergström I, Landgren B, Brinck J and Freyschuss B: Physical training preserves bone mineral density in postmenopausal women with forearm fractures and low bone mineral density. Osteoporos Int 19: 177-183, 2008.

18. Bonewald LF and Johnson ML: Osteocytes, mechanosensing and Wnt signaling. Bone 42: 606-615, 2008

19. Bocalini DS, Serra AJ, dos Santos L, Murad N and Levy RF: Strength training preserves the bone mineral density of postmenopausal women without hormone replacement therapy. J Aging Health 21: 519-527, 2009.
20. Hamilton CJ, Swan VJ and Jamal SA: The effects of exercise and physical activity participation on bone mass and geometry in postmenopausal women: a systematic review of pQCT studies. Osteoporos Int 21: 11-23, 2010.

21. Chinese Osteoporosis and Bone Mineral Association: Secondary osteoporosis diagnosis and treatment guidelines. Chinese J General Practitioners 5: 459-460, 2006 (In Chinese). 